\title{
Evaluation of the Micellization Mechanism of an Amphipathic Graft Copolymer with Enhanced Solubility of Ipriflavone
}

\author{
Satoshi Tanida, Tsuyoshi Kurokawa, Hideyuki Sato, Kazunori Kadota, and Yuichi Tozuka* \\ Osaka University of Pharmaceutical Sciences; 4-20-1 Nasahara, Takatsuki, Osaka 569-1094, Japan.
}

Received August 24, 2015; accepted November 5, 2015

The main purpose of this study was to investigate the solubilization enhancement properties of an amphipathic graft copolymer, Soluplus ${ }^{\circledR}$, on test compounds. Micellization of Soluplus ${ }^{\circledR}$ in solution was characterized by evaluating the changes in the surface activity, turbidity, and thermodynamic behavior. To assess the feasibility of Soluplus ${ }^{\circledR}$ as a polymeric carrier of solid dispersions, freeze-dried samples of ipriflavone were prepared, and the physicochemical properties of the carrier plus ipriflavone were evaluated in terms of solubility, dissolution, and crystallinity. The surface tension of the solution decreased depending on the polymer concentration, and gradual turbidity increase was observed. Isothermal titration calorimetry was used to measure the thermal reaction accompanying the micellization of Soluplus ${ }^{\circledR}$ and indicated that a colloidal micelle formation improved solubility. The prepared formulations, particularly at a ratio of ipriflavone:Soluplus ${ }^{\circledR}=1: 10(\mathrm{w} / \mathrm{w})$ exhibited a dramatically improved solubility of ipriflavone that was $c a$. 70-fold higher than that of untreated ipriflavone. The solubilization mechanism of Soluplus ${ }^{\circledR}$ was partially elucidated and suggested that its strategic application could improve the solubility of hydrophobic compounds.

Key words isothermal titration calorimetry; ipriflavone; amphipathic graft copolymer; solubility

Among the available technologies, the solid dispersion technique is commonly used as one of the most promising strategies for the enhancement of the solubility, dissolution rate, and bioavailability of poorly water-soluble compounds. A solid dispersion system can be defined as a distribution of active pharmaceutical ingredients as molecular amorphous particles and/or in the microcrystalline state into a carrier matrix-like polymer. ${ }^{1)}$ The physicochemical characteristics of polymeric carriers could dominate the pharmaceutical properties of a solid dispersion system. ${ }^{2,3)}$ Based on the characteristic properties of a polymeric carrier, a number of solid dispersion systems possessing various functional properties such as sustained release, $\mathrm{pH}$-dependent release, self-emulsifying properties, and site-specific release have been developed. ${ }^{4-7)}$ In particular, amphiphilic polymers have been found to be beneficial for the enhancement of not only solubility but also of the oral bioavailability of poorly water-soluble compounds because they form micellar structures that contain the compound. ${ }^{6,8)}$

Soluplus $^{\circledR}$, a graft copolymer consisting of polyvinyl caprolactam, polyvinyl acetate, and polyethyleneglycol, has been applied to the design of solid-dispersion formulations with poorly water-soluble substances to improve their solubility and bioavailability. ${ }^{910)}$ The copolymer exhibits an amphipathic property attributed to its hydrophilic and hydrophobic residues, which could contribute to the formation of micelles in water. Unlike conventional polymers such as polyvinylpyrrolidone and hydroxypropyl methylcellulose, Soluplus ${ }^{\circledR}$ is bifunctional and can act as both a polymeric carrier of solid dispersions and as a solubilizer through the formation of micelles in water, ${ }^{11-13)}$ which could be useful for the production of highly water-soluble formulations. Furthermore, the polymer has an inherent binding site that allows hydrogen bonding and/or van der Waal's interactions with drug molecules, which can lead to the stabilization of the drug molecule in the matrix. ${ }^{14)}$ Considering this background, Soluplus ${ }^{\circledR}$ has been applied to solid dispersion formulations prepared by freeze-drying, spray- drying, and melt-extrusion techniques to enhance the solubility and bioavailability of poorly water-soluble compounds. ${ }^{15-17)}$ However, the solubilizing mechanism of Soluplus ${ }^{\circledR}$ ascribed to micellization has yet to be elucidated. The self-organization of surfactants and amphiphilic polymers into micelles has been widely researched using many analytical methods, including the measurement of surface tension, Fourier transform infrared spectroscopy, relaxation studies using ${ }^{1} \mathrm{H}$ nuclear magnetic resonance, fluorescence spectroscopy, and isothermal titration calorimetry (ITC). ${ }^{18-22)}$ The ITC method has been used to characterize micellar-based systems because the detailed thermodynamic behavior of micellization could be determined by a single experiment, without the need of a probe. ${ }^{22,23)}$

The main objective of this study was to evaluate the micellization properties of Soluplus ${ }^{\circledR}$ in water and its solubility enhancement effect on an amorphous solid dispersion (ASD) system. Ipriflavone was selected as a model compound of a poorly water-soluble compound, which is generally used in the treatment of post-menopausal and senile osteoporosis by oral administration. ${ }^{24}$ The molecule is highly lipophilic with extremely low solubility in water and is metabolized by firstpass metabolism, which leads to the low bioavailability and variation in blood concentration. ${ }^{25,26)}$ For the characterization of the micellization properties in water, the surface activity, turbidity, and thermodynamic behavior were analyzed. The ASDs of ipriflavone were also evaluated based on their physicochemical properties, including solubility, dissolution behavior, and crystallinity.

\section{Experimental}

Chemicals Ipriflavone was supplied from Towa Pharmaceutical Co., Ltd. (Osaka, Japan) (Fig. 1). Soluplus ${ }^{\circledR}$ was obtained from BASF Japan Ltd. (Tokyo, Japan). All other chemicals and solvents used were reagent grade or HPLC grade.

Surface Tension The surface tension of Soluplus ${ }^{\circledR}$ aqueous solution was measured using an online tensiometer, 


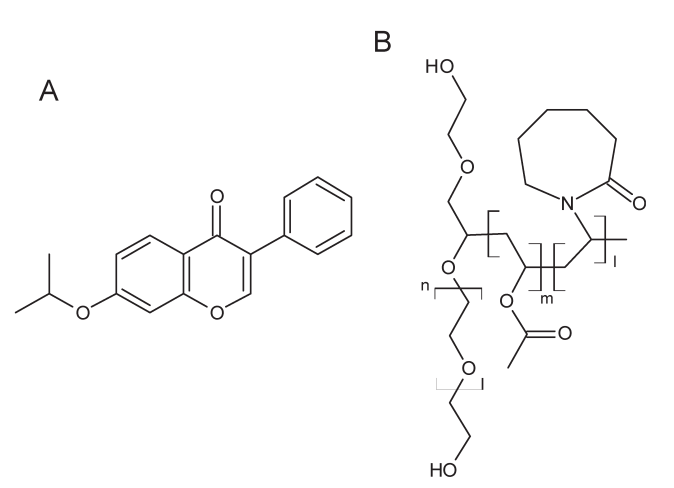

Fig. 1. Chemical Structures of (A) Ipriflavone and (B) Soluplus ${ }^{\circledR}$

SITA Science Line t60 (EKO Instruments Co., Ltd., Japan) at $25^{\circ} \mathrm{C}$. This tensionmeter analyzes the whole dynamic range of surface tension measurements based on the bubble pressure. A long bubble time $(1000 \mathrm{~ms})$ was selected to measure a semi-static condition. The measurements were performed in triplicate.

Turbidity The turbidity of Soluplus ${ }^{\circledR}$ aqueous solution over a specified concentration range $(0.01-100 \mathrm{mg} / \mathrm{mL})$ was analyzed by using a UV-Vis spectrophotometer (U-2900, Hitachi, Ltd., Japan) at $500 \mathrm{~nm}$. The measurements were performed in triplicate.

Dynamic Light Scattering (DLS) The particle size distributions of micelle-like structures were measured using DLS and a Nanotrac UPA UPA-UT151 (MicrotracBEL Corp., Osaka). Soluplus ${ }^{\circledR}$ was dissolved in distilled water, and the measurements of particle size distribution were performed in triplicate.

Isothermal Titration Calorimetry The thermodynamic behavior of Soluplus ${ }^{\circledR}$ aqueous solution was evaluated using a MicroCal iTC200 (Malvern Instrument Ltd., U.K.) to confirm the micellization process. The calorimetric titration was performed by injecting a polymer solution $(50 \mathrm{mg} / \mathrm{mL})$ into water at $25^{\circ} \mathrm{C}$. The sample cell $(300 \mu \mathrm{L})$ was filled with water, and the polymer solution was injected from the syringe into the sample cell under the agitation of $750 \mathrm{rpm}$. The time interval of each injection $(1 \mu \mathrm{L})$ was $120 \mathrm{~s}$. The thermodynamic changes within the sample cell were measured, and the changes of enthalpy depending on the concentration of Soluplus ${ }^{\circledR}$ were assessed. ${ }^{22)}$ All data were obtained and analyzed by using Origin iTC200 software (Malvern Instrument Ltd.).

Preparation of Ipriflavone ASDs Ipriflavone (250 mg) and different amounts of Soluplus ${ }^{\circledR}(0,500,1250$, or $2500 \mathrm{mg})$ were completely dissolved in $100 \mathrm{~mL}$ of tert-butyl alcohol. These solutions were frozen at $-40^{\circ} \mathrm{C}$ and then the frozen samples were freeze-dried using an FDU-830 freeze dryer (Tokyo Rikakikai Co., Ltd., Tokyo, Japan) below $30 \mathrm{~Pa}$ for 24h. The physical mixtures (PMs) were also prepared at the same ratio of ASD formulations.

Solubility Study The solubilities of ipriflavone samples were evaluated on untreated ipriflavone, PMs, and ASDs. Samples containing $50 \mathrm{mg}$ of ipriflavone were added to $25 \mathrm{~mL}$ of distilled water with specific concentrations of Soluplus ${ }^{\circledR}$ (PMs: 0.001, 0.01, 0.1, 1, 5, 10, 20, $100 \mathrm{mg} / \mathrm{mL}$, ASDs: 4, 10, $20 \mathrm{mg} / \mathrm{mL})$ and incubated at $37^{\circ} \mathrm{C}$ with agitation at 100 strokes/ min (ML-10, TAITEC Co., Ltd., Saitama, Japan) for 4 and $24 \mathrm{~h}$. These samples were centrifuged at $15000 \times \mathbf{g}$, and the supernatants were filtered through a membrane filter $(0.2 \mu \mathrm{m}$,

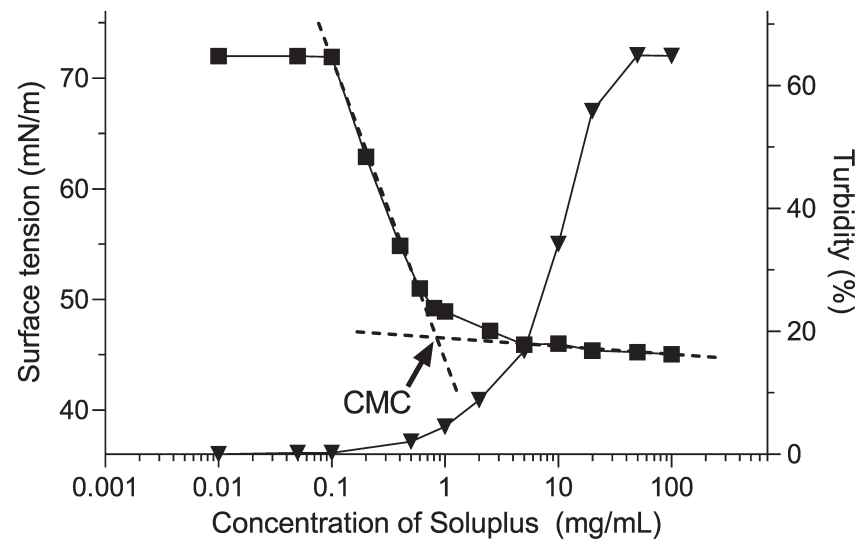

Fig. 2. Changes in the Surface Tension ( $\square$ ) and Turbidity ( $\boldsymbol{\nabla})$ of Soluplus ${ }^{\circledR}$ Aqueous Solution as a Function of the Soluplus ${ }^{\circledR}$ Concentration in Distilled Water

polyvinylidene difluoride (PVDF)). The solubility of ipriflavone was determined using an HPLC (SPD-10A, Shimadzu Co., Ltd., Kyoto, Japan) under the following conditions: pump, LC-10AD; detector, SPD-10A; column, COSMOSIL (4.6 mm $\phi \times 150 \mathrm{~mm}$; Nacalai Tesque Co., Ltd., Kyoto, Japan); column temperature, $40^{\circ} \mathrm{C}$; wavelength, $254 \mathrm{~nm}$; injection volume, $10 \mu \mathrm{L}$, and flow rate, $1.0 \mathrm{~mL} / \mathrm{min}$. The mobile phase was acetonitrile-water $(60: 40)$.

Dissolution Test The dissolution behaviors of the ipriflavone samples, including untreated ipriflavone, PM (ipriflavone: Soluplus ${ }^{\circledR}=1: 10$ ), and ASD (ipriflavone: Soluplus ${ }^{\circledR}=1: 10$ ), were evaluated using the dissolution test apparatus NTR-8000AC (Toyama Sangyo, Osaka, Japan). Samples equivalent to $5.0 \mathrm{mg}$ of ipriflavone were weighed $(5.0 \mathrm{mg}$ of untreated ipriflavone, $55.0 \mathrm{mg}$ of PM, or $55.0 \mathrm{mg}$ of ASD) and added into the dissolution medium $(900 \mathrm{~mL}$ of distilled water maintained at $37 \pm 0.5^{\circ} \mathrm{C}$ ) with a paddle speed of $50 \mathrm{rpm}$. At specific time points $(5,10,15,30,45,60,120 \mathrm{~min}), 3 \mathrm{~mL}$ of the test solutions were collected and centrifuged at $15000 \times \boldsymbol{g}$, and supernatants were filtered through a membrane filter $(0.2 \mu \mathrm{m}, \mathrm{PVDF})$. The dissolution amounts of ipriflavone were determined using HPLC, as described in Solubility Study section.

Powder X-Ray Diffraction (PXRD) The PXRD patterns were collected using a Rigaku Miniflex 600 powder X-ray diffractometer (Rigaku Corporation, Tokyo, Japan). The scanning rate was $4 \%$ min over a $2 \theta$ range of $5-35^{\circ}$ at a step size of $0.02^{\circ}$.

\section{Results and Discussion}

Figure 2 shows the changes in surface tension and turbidity as a function of the concentration of Soluplus ${ }^{\circledR}$ aqueous solution. The surface tension of Soluplus ${ }^{\circledR}$ aqueous solution gradually decreased and reached a plateau at around $10 \mathrm{mg} / \mathrm{mL}$, which was attributed to the saturation of the water surface by the polymer molecules (Fig. 2). The surface tension decreased to $45.3 \mathrm{mN} / \mathrm{m}$, which indicated a weak surface active property and the potential for micellization. The calculated critical micelle concentration (CMC) of Soluplus ${ }^{\circledR}$ was $0.82 \mathrm{mg} / \mathrm{mL}$. The solution became gradually cloudy as the concentration of Soluplus ${ }^{\circledR}$ increased above the CMC, which indicated the formation of a colloidal micelle structure that was found to be $c a .60 \mathrm{~nm}$ using a DLS method. The micellar structure had 
(A)

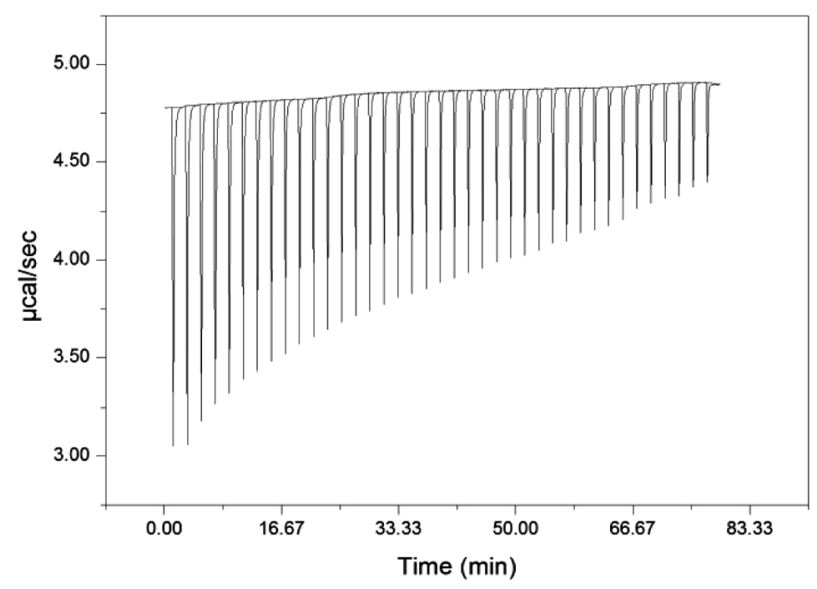

(B)

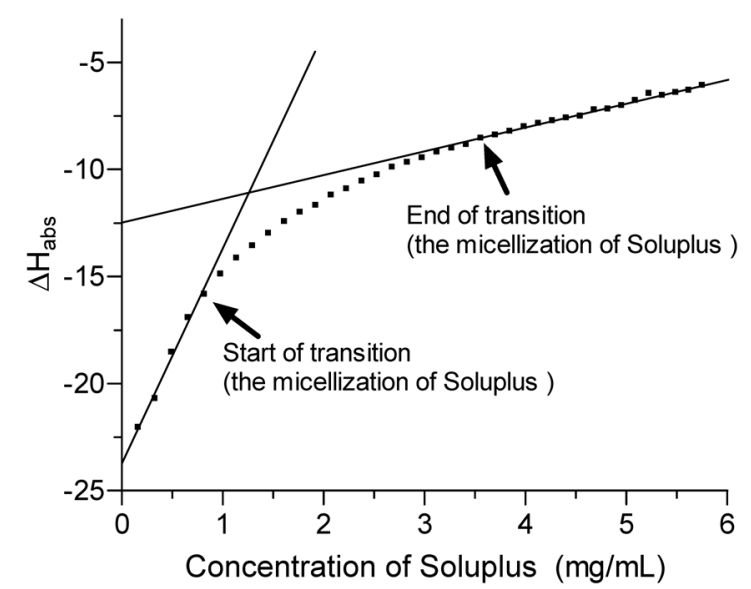

Fig. 3. Data Obtained from the ITC Analysis

(A) Exothermic heat release on injection of Soluplus ${ }^{\circledR}$ solution into water. (B) Integrated heat data from the ITC analysis. ST and ET, which represent the start of transition and end of micellization, respectively.

not changed after $24 \mathrm{~h}$ of storage under ambient conditions. Soluplus ${ }^{\circledR}$ was able to form micelles because of the amphipathic property conferred by the polyethylene glycol backbone as the hydrophilic part and vinylcaprolactam/vinyl acetate side chains as the lipophilic structure in aqueous solution; this property has been shown to improve the solubility and dissolution behavior of co-existent drugs. ${ }^{11,13)}$

To gain further knowledge of the micellization properties, the thermodynamic changes of Soluplus ${ }^{\circledR}$ as a function of concentration were analyzed by ITC. ITC is a powerful tool for obtaining calorimetric information about the self-organization of surfactants into micelles and can quantify the enthalpy derived from the micellization of a surfactant and amphiphilic polymer in a single experiment without the necessity of a probe. $^{22,23,27)}$ In the case of conventional surfactants such as sodium dodecyl sulfate or cetyltrimethylammonium bromide, the ITC thermograms display sigmoidal curves and three concentration ranges: (i) the dissociation range of micelles with repeated dilution of a titrated solution, (ii) the micellization range of surfactant molecules, and (iii) the dilution of micelles. $^{22,28)}$

In (i), an exothermic reaction could occur because of the breakup of micelles into unimers and dilution of the resultant unimers when the final concentration of surfactant was below the CMC in the sample cell. In (ii), a clear decrease in the endothermic reaction was observed because of the decline in the dissociation of micelles and the micellization of unimers when additional micellar solution was added to the sample cell. In (iii), a small thermal reaction was observed that was caused by the dilution of the micellar solution when more micellar solution was injected above the CMC. Although changes in enthalpy caused by thermal reaction were observed depending on the concentration of Soluplus ${ }^{\circledR}$ in the present work, the ITC thermogram demonstrated a non-sigmoidal shape (Fig. 3). The non-sigmoidal curve could reflect the abrupt change in the structure of the polymer molecule after a thermal reaction at a very low concentration of Soluplus ${ }^{\circledR}$. Under this experimental condition, thermodynamic reactions derived from the phase of (ii) and (iii) may be observed. The non-sigmoidal thermograms were analyzed according to a method that has been re- ported by many authors ${ }^{23,29,30)}$ and enables a rough estimation to be made of the start of transition (ST) and end of transition (ET) corresponding to the start and end of the micellization process. As shown in Fig. 3B, the calculated values of ST and ET were $0.82 \mathrm{mg} / \mathrm{mL}$ and $3.56 \mathrm{mg} / \mathrm{mL}$, respectively. The ST was almost the same value of the CMC calculated from the surface tension analysis, a finding that could also support the hypothesis that the micellar structure of Soluplus ${ }^{\circledR}$ leading to the solubilization of ipriflavone was formed above the CMC and ST value.

As shown in Fig. 4, even in physical mixture samples, the addition of Soluplus ${ }^{\circledR}$ to ipriflavone significantly enhanced the solubility in proportion to the concentration of the polymer that corresponded to the decrease in surface tension and increase in turbidity, which suggests that the inclusion of ipriflavone into the micellar structure occurred above the CMC of Soluplus ${ }^{\circledR}$. Moreover, the micellization of Soluplus ${ }^{\circledR}$ led to highly stable saturation state of ipriflavone, which was maintained for at least $24 \mathrm{~h}$ (Fig. 5A). The interactions and complex-forming abilities of polymeric carriers with drug molecules would be critical for the enhancement of solubility and conferring supersaturation properties. Soluplus ${ }^{\circledR}$ has multiple binding sites on the lipophilic residues created by grafting vinyl acetate and vinylcaprolactam moieties, which can achieve stronger entanglement with hydrophobic compounds. ${ }^{31)}$ As reported in the literature, a lower dose of a surface active compound could increase the absorption efficacy of a poorly absorbable drug by altering the permeability of the cell membrane. ${ }^{32,33)}$ In addition to the solubility enhancement based on micellization, the absorption enhancement effect of Soluplus ${ }^{\circledR}$ could be beneficial for the absorption process and improvement of oral bioavailability. From these findings, the application of Soluplus ${ }^{\circledR}$ as a polymeric carrier for a solid dispersion system could have the potential for the development of highly soluble and absorbable formulations to achieve efficacious pharmaceutical effects.

For the evaluation of the feasibility of Soluplus ${ }^{\circledR}$ as a polymeric carrier of solid dispersions, the ASD of ipriflavone was prepared using the freeze-drying technique, and the physicochemical properties of the carrier and compound 
were assessed. The solubility enhancement effect of the ASD system that was based on the micellization of Soluplus ${ }^{\circledR}$ was estimated in a solubility study (Fig. 5). The solubility of untreated ipriflavone has extremely low solubility in water $(1.0 \mu \mathrm{g} / \mathrm{mL})$. On the other hand, PMs and ASDs dramatically enhanced the solubility of ipriflavone, particularly in the ASD (ipriflavone: Soluplus ${ }^{\circledR}=1: 10$ ), $c a$. 70-fold higher than that of untreated ipriflavone. As compared with the PM samples, the additional enhancement of the solubility with ASD system could be achieved by the amorphization of ipriflavone and formation of a solid dispersion. This supersaturated state was maintained for $\geq 24 \mathrm{~h}$ because of the formation of highly stable micellar structures. Dissolution studies were also performed using untreated ipriflavone, PM, and ASD (ipriflavone:Soluplus ${ }^{\circledR}=1: 10$ ) to evaluate the drug release from the ipriflavone samples. The dissolution amount of untreated

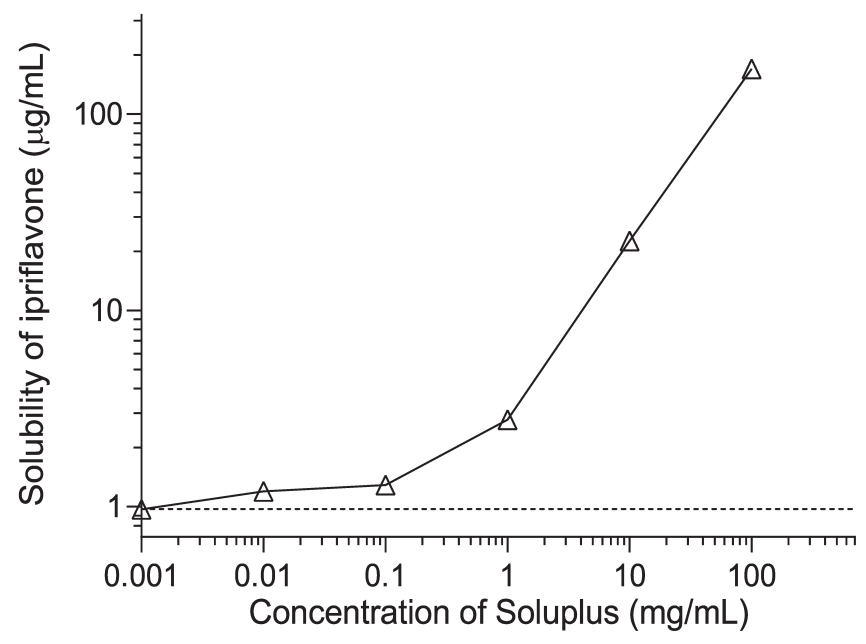

Fig. 4. Relationship between Soluplus ${ }^{\circledR}$ Concentration and the Solubility of Ipriflavone When the Simply Blended Powder Was Incubated for $24 \mathrm{~h}$ in Water

The dashed line represents the saturation solubility of ipriflavone in water $(1.0 \mu \mathrm{g} / \mathrm{mL})$.

\section{(A)}

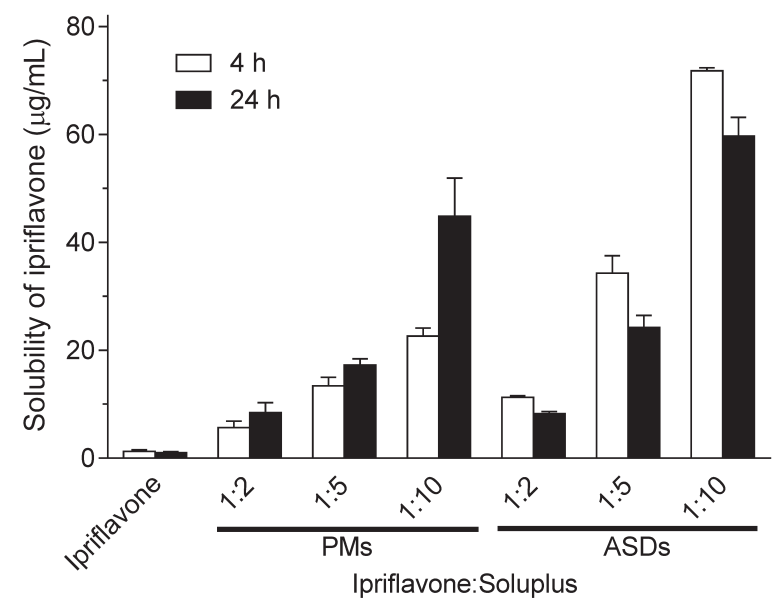

ipriflavone after a 120 -min test was negligible because of the poorly water-soluble property. In the case of PM consisting of ipriflavone: Soluplus ${ }^{\circledR}=1: 10$, the dissolution rate and dissolved amount of ipriflavone were slightly higher than that of untreated ipriflavone because of the solubilizing effect of Soluplus ${ }^{\circledR}$ in water, which corresponded to the result from the solubility study of PM (Fig. 4). Moreover, the dissolution test confirmed that the ASD formulation had a better dissolution rate than that of PM (Fig. 5B). To examine the crystallinity of ipriflavone, untreated ipriflavone, PM, and ASD samples were investigated by PXRD analysis. As shown in Fig. 6, untreated ipriflavone and PMs exhibited diffraction peaks of crystalline ipriflavone. The diffraction peaks indicating crystalline of ipriflavone gradually decreased as the ratio of Soluplus in PMs increased. ASD (ipriflavone: Soluplus ${ }^{\circledR}=1: 2$ ) exhibited weak diffraction peaks similar to the diffraction pattern of untreated ipriflavone, which indicated the residual crystalline phase of ipriflavone in the formulation. On the other hand, there were no diffraction peaks when Soluplus ${ }^{\circledR}$ and the ASDs (ipriflavone: Soluplus ${ }^{\circledR}=1: 5,1: 10$ ) were analyzed. The higher drug loading in the solid dispersion formulation

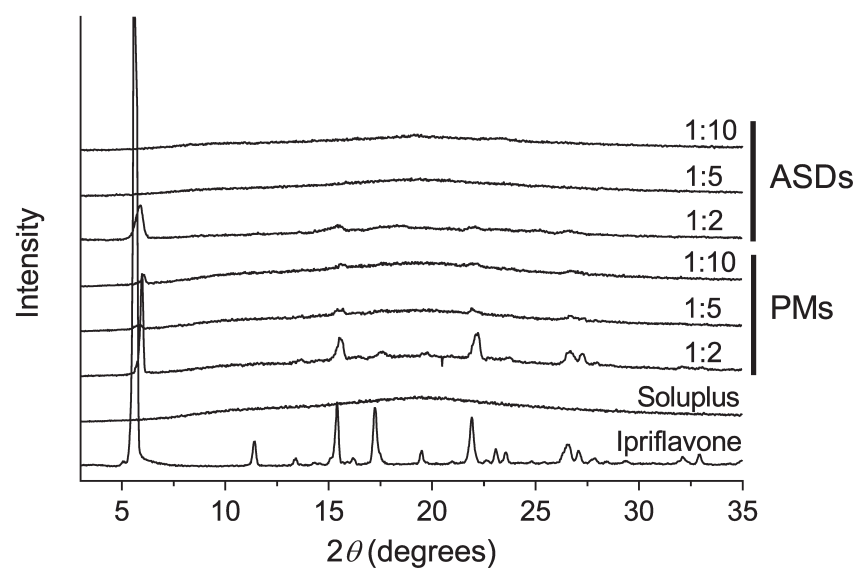

Fig. 6. PXRD Patterns of Ipriflavone Samples
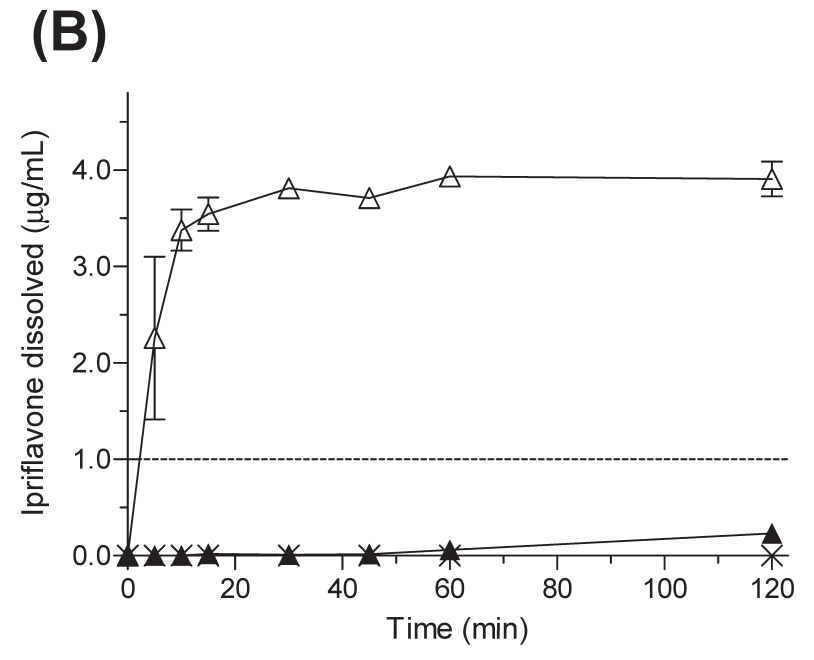

Fig. 5. (A) The Solubility of Ipriflavone Samples, Including Untreated Ipriflavone, PMs Samples (Ipriflavone: Soluplus ${ }^{\mathbb{B}}=1: 2,1: 5,1: 10$ ), and ASD Samples (Ipriflavone:Soluplus $\left.{ }^{\circledR}=1: 2,1: 5,1: 10\right)$ Prepared by Freeze-Drying; (B) Dissolution Profiles of Untreated Ipriflavone $(\times$ ), PM (Ipriflavone: Soluplus $\left.{ }^{\circledR}=1: 10\right)(\boldsymbol{\Lambda})$, and Freeze-Dried Formulation (Ipriflavone: Soluplus $\left.{ }^{\circledR}=1: 10\right)\left(\triangle\right.$ ) in Distilled Water at $37^{\circ} \mathrm{C}$

The dashed line represents the saturation solubility of ipriflavone in water $(1.0 \mu \mathrm{g} / \mathrm{mL})$. Each bar represents the mean \pm S.E. of three experiments. 
could cause crystallization, precipitation, and/or phase separation of drug compound during the manufacturing process or storage because of the lack of sufficient interaction sites on the polymers for stabilization of the amorphous state. Generally, solid dispersions improve solubility, dissolution rates, and bioavailability because of amorphization and particle size reduction of inclusion drugs, which increases wettability and dispersibility derived from hydrophilic polymers. ${ }^{34)}$ The ASD system prepared in the present study may have improved these physicochemical properties of ipriflavone and enhanced the solubility and dissolution rate. In addition to these physicochemical characteristics, the ASD system had a solubilizing effect that was achieved by micellization. The strategic application of Soluplus $^{\circledR}$ as a polymeric carrier of solid dispersions may improve the solubility of hydrophobic compounds.

In this study, the mechanism of solubilization based on the micellization of Soluplus ${ }^{\circledR}$ was partially proven with respect to the surface tension, turbidity, and thermodynamic behavior. Using the ITC technique, the micellization behavior of Soluplus ${ }^{\circledR}$ was confirmed thermodynamically, which could also support the hypothesis that the micellar structure of Soluplus ${ }^{\circledR}$ leading to the solubilization of ipriflavone was formed above the CMC. Based on the micellization properties of Soluplus ${ }^{\circledR}$, a highly soluble ASD system was developed using the freezedrying technique to improve the solubility and dissolution behavior of ipriflavone. The ASD formulations, particularly at a ratio of ipriflavone:Soluplus ${ }^{\circledR}=1: 10(\mathrm{w} / \mathrm{w})$, dramatically improved the solubility of ipriflavone $c a$. 70-fold higher than that of untreated ipriflavone because of the formation of micelles incorporating ipriflavone and amorphization of the drug. In conclusion, the strategic application of Soluplus ${ }^{\circledR}$ could be beneficial for ipriflavone and other insoluble drugs and enable successful development of highly water-soluble ASD formulations.

Acknowledgments We would like to thank Towa Pharmaceutical Co., Ltd. and BASF JAPAN Ltd. for their kind gift of ipriflavone and Soluplus ${ }^{\circledR}$, respectively. The authors express appreciation to EKO Instruments Co., Ltd. for the measurement of the surface tension. We also wish to thank Dr. Koji Tomoo, Osaka University of Pharmaceutical Sciences, for the technical support for the ITC analysis.

Conflict of Interest The authors declare no conflict of interest.

\section{References}

1) Chiou W. L., Riegelman S., J. Pharm. Sci., 60, 1281-1302 (1971).
2) Ueda H., Aikawa S., Kashima Y., Kikuchi J., Ida Y., Tanino T.,
Kadota K., Tozuka Y., J. Pharm. Sci., 103, 2829-2838 (2014).
3) Ueda H., Wakabayashi S., Kikuchi J., Ida Y., Kadota K., Tozuka Y.,
Mol. Pharm., 12, 1050-1061 (2015).
4) Li J., Lee I. W., Shin G. H., Chen X., Park H. J., Eur. J. Pharm.
Biopharm., 94, 322-332 (2015).

5) Zecevic D. E., Meier R., Daniels R., Wagner K. G., Eur. J. Pharm. Biopharm., 87, 264-270 (2014).

6) Yadava S. K., Naik J. B., Patil J. S., Mokale V. J., Singh R., Colloid. Surf. A, 481, 63-71 (2015).

7) Hecq J., Siepmann F., Siepmann J., Amighi K., Goole J., Drug Dev. Ind. Pharm., 2015, 1-8 (2015).

8) Tabatabaei Rezael S. J., Abandansari H. S., Nabid M. R., Niknejad H., J. Colloid. Interface Sci., 425, 27-35 (2014).

9) Ha E. S., Baek I. H., Cho W., Hwang S. J., Kim M. S., Chem. Pharm. Bull., 62, 545-551 (2014).

10) Han S. D., Jung S. W., Jang S. W., Jung H. J., Son M., Kim B. M., Kang M. J., Chem. Pharm. Bull., 63, 295-299 (2015).

11) Dian L., Yu E., Chen X., Wen X., Zhang Z., Qin L., Wang Q., Li G., Wu C., Nanoscale Res. Lett., 9, 684 (2014).

12) Nagy Z. K., Balogh A., Vajna B., Farkas A., Patyi G., Kramarics A., Marosi G., J. Pharm. Sci., 101, 322-332 (2012).

13) Yu H., Xia D., Zhu Q., Zhu C., Chen D., Gan Y., Eur. J. Pharm. Biopharm., 85 (3 Pt B), 1325-1336 (2013).

14) Alonzo D. E., Zhang G. G., Zhou D., Gao Y., Taylor L. S., Pharm. Res., 27, 608-618 (2010).

15) Alshahrani S. M., Lu W., Park J. B., Morott J. T., Alsulays B. B., Majumdar S., Langley N., Kolter K., Gryczke A., Repka M. A., AAPS PharmSciTech, 16, 824-834 (2015).

16) Homayouni A., Sadeghi F., Nokhodchi A., Varshosaz J., Afrasiabi Garekani H., Iran J. Pharm. Res., 14, 35-50 (2015).

17) Jin X., Zhou B., Xue L., San W., Biomed. Pharmacother., 69, 388 395 (2015).

18) Ma J. H., Guo C., Tang Y. L., Liu H. Z., Langmuir, 23, 9596-9605 (2007).

19) Su Y. L., Wang J., Liu H. Z., J. Colloid. Interface Sci., 251, 417-423 (2002).

20) Zhang S., Li N., Zheng L., Li X., Gao Y., Yu L., J. Phys. Chem. B, 112, 10228-10233 (2008).

21) Zhang J., Tozuka Y., Uchiyama H., Higashi K., Moribe K., Takeuchi H., Yamamoto K., J. Pharm. Sci., 100, 4421-4431 (2011).

22) Bouchemal K., Agnely F., Koffi A., Ponchel G., J. Colloid. Interface Sci., 338, 169-176 (2009).

23) Bouchemal K., Agnely F., Koffi A., Djabourov M., Ponchel G., $J$. Mol. Recognit., 23, 335-342 (2010).

24) Head K. A., Altern. Med. Rev., 4, 10-22 (1999).

25) Ferenc L., Istvan S., Acta Pharm. Hung., 65, 219-222 (1995).

26) Ferenc L., Istvan S., Acta Pharm. Hung., 65, 215-218 (1995).

27) Al-Obaidi H., Ke P., Brocchini S., Buckton G., Int. J. Pharm., 419. 20-27 (2011).

28) Beyer K., Leine D., Blume A., Colloid. Surf. B Biointerfaces, 49, 31-39 (2006).

29) Roques C., Bouchemal K., Ponchel G., Fromes Y., Fattal E., J. Control. Release, 138, 71-77 (2009).

30) Klijn J. E., Kevelam J., Engberts J. B., J. Colloid. Interface Sci., 226, 76-82 (2000).

31) Guo Z., Lu M., Li Y., Pang H., Lin L., Liu X., Wu C., J. Pharm. Pharmacol., 66, 285-296 (2014).

32) Aungst B. J., J. Pharm. Sci., 89, 429-442 (2000).

33) Linn M., Collnot E. M., Djuric D., Hempel K., Fabian E., Kolter K., Lehr C. M., Eur. J. Pharm. Sci., 45, 336-343 (2012).

34) Alam M. A., Ali R., Al-Jenoobi F. I., Al-Mohizea A. M., Expert Opin. Drug Deliv., 9, 1419-1440 (2012). 